\title{
Spielräume für eine gerechtere Steuerpolitik in den OECD-Ländern
}

Die zunehmende Schieflage in der Einkommens- und Vermögensverteilung der meisten OECD-Länder hat fast überall zu Forderungen nach steuerpolitischen Korrekturen geführt. Allerdings sehen sich diese Forderungen dem weit verbreiteten Argument ausgesetzt, mehr Steuergerechtigkeit sei nur um den Preis erheblicher Wachstums- und Beschäftigungsverluste zu haben. Doch wie sehen die steuerpolitischen Trends und die aktuellen Entwicklungen überhaupt aus? Muss man wirklich mit negativen ökonomischen Auswirkungen einer gerechteren Steuerpolitik rechnen? Könnte eine stärkere Umverteilung aus makroökonomischer Sicht nicht sogar vorteilhaft sein? Welche Schlussfolgerungen ergeben sich für die Steuerpolitik auf nationaler und internationaler Ebene? ${ }^{1}$

SARAH GODAR, CHRISTOPH PAETZ, ACHIM TRUGER

\section{Problemstellung}

Die Ungleichheit in der Einkommens- und Vermögensverteilung ist in den letzten Jahrzehnten OECD-weit erheblich gestiegen. Gleichzeitig sehen sich viele Staaten nach der Großen Rezession mit großen Konsolidierungslasten konfrontiert. Daher werden seit geraumer Zeit weithin Forderungen nach progressiven Steuerreformen laut. Allerdings ist das dominierende ökonomische Argument gegen solche Reformen, dass sie sich nachteilig auf Wachstum und Beschäftigung auswirkten und zu einem Anstieg der Steuerumgehung führten. Der vorliegende Aufsatz liefert eine kritische Bewertung solcher Standardargumente und ergänzt sie um eine gesamtwirtschaftliche Perspektive. Es zeigt sich, dass die Spielräume der nationalen Regierungen für eine progressive Steuerpolitik und für Einnahmenerhöhungen viel größer sind als vielfach behauptet.

Abschnitt 2 gibt zunächst einen Überblick über die regressiven Steuertrends seit den 1980er Jahren. Im Anschluss daran wird gezeigt, dass trotz einiger Änderungen und politischer Vorschläge keine Anzeichen für eine umfassende Trendwende vorliegen, da dieser nach vorherrschender Auffassung ein Zielkonflikt zwischen Gerechtigkeit und Effizienz im Wege steht (3). Abschnitt 4 stellt die ökonomischen Standardargumente, die häufig gegen progressive Steuerreformen ins Feld geführt werden, auf den Prüfstand. Schließlich wird die Analyse um eine makroökonomische
Perspektive ergänzt (5), bevor abschließend Schlussfolgerungen für die nationale und internationale Steuerpolitik gezogen werden (6).

\section{Steuergerechtigkeit seit den 1980er Jahren auf dem Rückzug}

Traditionell lag der verteilungspolitischen Zielsetzung der Steuerpolitik das Leistungsfähigkeitsprinzip zugrunde, wonach (a) alle Einkommen umfassend und gleichmäßig zu besteuern, d. h. Steuerprivilegien für bestimmte Einkommensarten zu vermeiden sind (synthetische Einkommensbesteuerung) und (b) eine starke Umverteilung,

1 Der Aufsatz basiert größtenteils auf Ergebnissen des Workpackage "Redistributive Policies" innerhalb des Global Labour University-Forschungsprojektes „Combating Inequality", das von der Hans-Böckler-Stiftung finanziert wurde (http://www.global-labour-university.org/353.html). Wir danken Alexander Gallas, Hansjörg Herr und Christoph Scherrer für sehr hilfreiche Kommentare zu einer früheren englischsprachigen Version des Aufsatzes. Zwei anonymen Gutachtern der WSI-Mitteilungen sind wir ebenfalls zu Dank verpflichtet. Für alle verbleibenden Fehler und Unklarheiten sind wir allein verantwortlich. 
ABB. 1

\section{Entwicklung von Unternehmenssteueraufkommen, Gewinnquote und Unternehmenssteuersätzen}

Unternehmenssteueraufkommen in Prozent des BIP (linke Achse)

- Nominale Unternehmenssteuersätze in Prozent (rechte Achse)

-.- Gewinnquote in Prozent des BIP (rechte Achse)

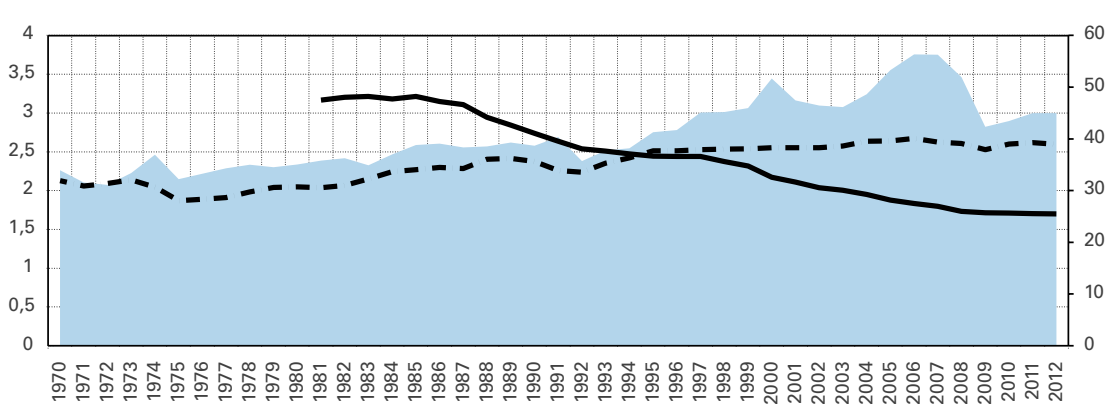

Quelle: OECD (2014a) gerückt und haben für Kapitalerträge natürlicher Personen Pauschalsteuern eingeführt, während die progressive Besteuerung im Bereich der Arbeitseinkommen beibehalten wurde. In vielen OECD-Ländern (z. B. Schweden, Finnland, Österreich, Deutschland, Spanien, Irland und Japan) sind bestimmte Arten von Kapitalerträgen natürlicher Personen (z.B. Zinsen, Dividenden und Kapitalgewinne) aus der progressiven Einkommensbesteuerung ausgeschlossen (OECD 2013a; Deloitte 2013). Schratzenstaller (2004, S. 23) verweist auf einen allgemeinen Trend weg von der synthetischen Besteuerung hin zu einer Dualisierung der Einkommensteuer. Veräußerungsgewinne werden häufig mit einem niedrigeren Steuersatz besteuert. Darüber hinaus gibt es vielfältige Steuervergünstigungen für verschiedenste Arten von Kapitalerträgen (Deloitte 2013). Die maximale tarifliche Steuerbelastung von Dividenden ist seit 1981 deutlich gesunken (OECD 2013a).

Die Besteuerung der Unternehmensgewinne, gemessen anhand der tariflichen Unternehmenssteuersätze, unterliegt seit fast drei Jahrzehnten einem internationalen Wettlauf nach unten. In den Ländern, für die OECD-Daten seit 1981 verfügbar sind, ist der (ungewichtete) Durchschnitt der Steuersätze um mehr als 20 Prozentpunkte gesunken - von 47,5\% im Jahr 1981 auf nur noch 27,2\% im Jahr 2012. ${ }^{3}$

Allerdings spiegeln sich die sinkenden Steuersätze nicht auf den ersten Blick in den Einnahmen wider: Bis 2007 ist das Unternehmenssteueraufkommen in Prozent des Bruttoinlandsprodukt (BIP) in den meisten OECD-Ländern im Vergleich zu den 1970er und 1980er Jahren sogar gestiegen (Abbildung 1). Trotz des erheblichen Rückgangs in den Jahren 2008 und 2009 war das durchschnittliche Niveau im Jahr 2010 immer noch etwas höher als 30 bzw. 40 Jahre zuvor. Die wahrscheinlichste Ursache für die dynamische Aufkommensentwicklung dürfte im steigenden Anteil der Unternehmensgewinne am BIP zu suchen sein: Der rasante Gewinnanstieg hat den Effekt der sinkenden Steuersätze (über-)kompensiert (Devereux et al. 2004, S. 26). So ist die bereinigte Gewinnquote im OECD-Durchschnitt von ihrem Tiefpunkt von knapp $28 \%$ des BIP zu Faktorkosten im Jahr 1975 auf knapp 39\% und damit um 11 Prozentpunkte bzw. fast $39 \%$ gestiegen (Europäische Kommission 2014).

Auch die effektive Besteuerung von Privatvermögen ist in den letzten Jahrzehnten erheblich zurückgegangen: Obwohl seit 1970 das Verhältnis von privatem Vermögen zum Volkseinkommen in vielen OECD-Ländern deutlich gestie-

2 Vgl. Godar/Truger (2015a) für einen ausführlichen Überblick.

3 Kompliziertere Indikatoren für effektive Steuersätze, wie die sogenannten Effektiven Grenz-, bzw. Durchschnittssteuersätze (EMTR bzw. und EATR), die auf mikroökonomischen Investitionstheorien beruhen (Spengel et al. 2012), sowie die aggregierten impliziten Steuersätze, die von Eurostat berechnet werden (Europäische Kommission 2012, S. 257), zeigen im Großen und Ganzen ein ähnliches Bild. 
gen ist (Piketty/Zucman 2013), stagnierten die Einnahmen der OECD-Länder aus vermögensbezogenen Steuern in Relation zum BIP.

\section{Aktuelle steuerpolitische Trends und Empfehlungen}

\subsection{Aktuelle Trends}

Angesichts der wachsenden Ungleichheit und der angespannten Lage der öffentlichen Haushalte in vielen OECDLändern seit der Großen Rezession gibt es einige Anzeichen dafür, dass der Abwärtstrend bezüglich der progressiven Besteuerung zum Stillstand gekommen ist. ${ }^{4}$ In der Mehrzahl der OECD-Länder wurden die Spitzensteuersätze bei der Einkommensteuer nach der Finanzkrise erhöht (IWF 2013, S. 26.). Seitdem hat eine Reihe von Ländern auch ihre maximalen Steuersätze auf private Kapitalerträge erhöht. Auch hat sich seit der Wirtschaftskrise der durchschnittliche Unternehmensteuersatz stabilisiert (OECD 2014a). Belgien, Griechenland, Irland, Portugal, Spanien und Großbritannien haben zudem die Steuern auf Immobilienbesitz erhöht (Europäische Komission 2012, S. 29ff.; IWF 2013, S. 26).

Während die genannten Entwicklungen zweifellos in Richtung größerer Steuergerechtigkeit gehen, gibt es auch gravierende gegenläufige Maßnahmen: Viele Regierungen haben seit 2009 die Mehrwertsteuersätze angehoben (Europäische Kommission 2013a, S. 31; IWF 2013, S. 26). Darüber hinaus wurden zahlreiche andere indirekte Steuern erhöht. Laut Europäischer Kommission (2013a, S. 30) haben sich die Konsolidierungsmaßnahmen auf der Einnahmenseite seit 2009 stark auf Konsumsteuern konzentriert, die regressiver Natur sind, sodass insgesamt die Progressivität der Besteuerung sogar abgenommen haben wird.

\subsection{Aktuelle Empfehlungen}

In den letzten Jahren haben viele bedeutende internationale Institutionen Vorschläge unterbreitet, wie man mit Steuerreformen zur Haushaltskonsolidierung beitragen kann, ohne dabei die Disparität in der Einkommensverteilung stark zu vergrößern (ETUC 2010; Europäische Kommission 2012, 2013c; European ATTAC Network 2013; Europäischer Rat 2012; ILO 2011; IWF 2013; ITUC 2010; OECD 2012c, 2012d, 2013b; Tax Justice Network 2013; UNCTAD 2012). Während es einen weit verbreiteten Konsens hinsichtlich der Bekämpfung der Steuerhinterziehung, der Begrenzung der Steuervermeidung und der Einführung einer Finanztransaktionssteuer gibt, gehen die Meinungen auseinander, wenn es um deutlich darüber hinausgehende progressive Reformen geht. Die
Gewerkschaften, die International Labour Organization (ILO), die United Nations Conference on Trade and Development (UNCTAD) und einige NGOs stellen solche Forderungen. Die dominierenden internationalen Institutionen, wie die Europäische Kommission, der IWF und die OECD sind dagegen sehr zurückhaltend, wenn nicht sogar offen dagegen. ${ }^{5}$

Die gängige Meinung des IWF (2013) im Hinblick auf die einnahmeseitige Konsolidierung konzentriert sich auf die Ausweitung der Bemessungsgrundlage der Mehrwertsteuer sowie der persönlichen Einkommens- und Unternehmenssteuern, die Erhöhung regelmäßiger Abgaben auf Wohneigentum und die Ausweitung von Umweltsteuern. Ziel ist es, zusätzliche Einnahmen zu generieren, ohne die privaten Haushalte mit niedrigem Einkommen unverhältnismäßig zu belasten - diese Sichtweise wird von der OECD (2012c) geteilt. Um die regressiven Auswirkungen der vorgeschlagenen Steuerrechtsänderungen zu mildern, schlagen beide Institutionen die Einführung zusätzlicher Sozialtransfers vor.

Einer der am häufigsten wiederholten Vorschläge der OECD zur Förderung des Wachstums sowie zur Bekämpfung der Ungleichheit besteht darin, Steuerschlupflöcher, von denen vornehmlich Wohlhabende profitieren, zu schließen und deren Steuervergünstigungen zu verringern (OECD 2012c, S. 3). Nach Angaben der OECD verzerren höhere Steuern auf Immobilien und Verbrauch die ansonsten „optimalen“ Entscheidungen der Besteuerten am wenigsten. Zwar könnten sie zu höherer Ungleichheit führen, verursachen darüber hinaus aber die geringsten wirtschaftlichen Schäden. Die OECD (2012c, S. 3) kommt zu dem Schluss, dass gezielte Transfers die Schwere dieses Zielkonflikts reduzieren können. Ebenso lädt der Europäische Rat die Mitgliedstaaten ein, gegebenenfalls ihre Steuersysteme zu überprüfen, mit dem Ziel, sie effektiver und effizienter zu gestalten. Dabei geht es um die Beseitigung ungerechtfertigter Ausnahmen, die Ausweitung der Bemessungsgrundlage, die Verlagerung der Besteuerung weg vom Faktor Arbeit, die Steigerung der Effizienz zur Steuererhebung und die Bekämpfung der Steuerhinterziehung (Europäischer Rat 2012, S. 3).

Einige der vorgeschlagenen Maßnahmen könnten zu einer Verringerung der Einkommensungleichheit führen oder zeigen zumindest eine gewisse Besorgnis bezüglich der negativen Verteilungseffekte der Besteuerung. Jedoch plädieren die angeführten Institutionen nicht für grundlegendere Reformen: Die Erhöhung der Spitzensteuersätze bei der Einkommensteuer und der Unternehmenssteuersätze sowie allgemeine Steuern auf Vermögen stehen

4 Vgl. Godar/Truger (2015a) für einen ausführlichen Überblick.

5 Vgl. Godar/Truger (2015b) für einen ausführlichen Überblick. 
nicht auf der Tagesordnung. ${ }^{6}$ Offensichtlich besteht der Hauptgrund dieser Zurückhaltung im wahrgenommenen Zielkonflikt zwischen Steuergerechtigkeit und Effizienz. Die einfache Anhebung der marginalen Steuersätze auf hohe Einkommen werde nicht unbedingt viele zusätzliche Einnahmen bringen. Grund seien negative Wirkungen, insbesondere auf die Arbeitsintensität, die Karriereentscheidungen und Steuervermeidungsaktivitäten (OECD 2012d, S. 39).

\section{Standardargumente gegen progressive Besteuerung auf dem Prüfstand}

Als Standardargument gegen eine progressivere Besteuerung wird häufig angeführt, sie gehe mit negativen Anreizen einher und verstärke die Steuervermeidungsaktivitäten. ${ }^{7}$ Allerdings kann man auf der Basis der Standard-Literatur (z. B. Rosen/Gayer 2008; Salanié 2011) durchaus auch zu dem Schluss kommen, dass die befürchteten Effekte nicht sonderlich groß sind: Der Zielkonflikt zwischen Steuergerechtigkeit und Effizienz könnte gering sein. Darüber hinaus können Staatsausgaben, die mit den erzielten zusätzlichen Einnahmen finanziert werden, die negativen Auswirkungen der Besteuerung auf Produktion und Beschäftigung ausgleichen oder sogar überkompensieren.

\subsection{Negative Wirkungen auf die Entscheidungen der privaten Haushalte?}

Bezüglich der privaten Haushalte beziehen sich die wichtigsten diskutierten negativen Anreizwirkungen auf Arbeitsangebot, Ersparnisbildung und, seit Kurzem, auch auf Steuervermeidung bzw. -flucht. ${ }^{8}$ Das typische Argument gegen eine progressivere Einkommensbesteuerung ist, dass Steuern den Nettoreallohn senken und daher die Arbeitsanreize reduzieren. Theoretisch ist der Gesamteffekt auf das Arbeitsangebot jedoch nicht eindeutig (Salanié 2011, S. 18). Da in der Regel die Notwendigkeit besteht, den Lebensunterhalt durch Arbeit zu bestreiten, und Arbeitsmarktentscheidungen stark von sozialen Normen geprägt sind, erscheint die Vorstellung, dass Menschen über ihre Beteiligung am Arbeitsmarkt in Bezug auf die Einkommensteuer entscheiden, insgesamt wenig überzeugend.

Empirisch hat sich das Arbeitsangebot als eher unelastisch erwiesen. Evers et al. (2008) untersuchen in einer Metastudie die empirischen Schätzungen der Lohnelastizität des Arbeitsangebots. Der Mittelwert der geschätzten Elastizitäten für das Arbeitsangebot der Männer liegt bei 0,07 und der Median liegt bei 0,08. Die entsprechenden Werte für Frauen sind - ohne die Berücksichtigung von Ausreißern - 0,43 und 0,27 bzw. 0,34 und 0,26 (ebd., S. 32). Dies bedeutet, dass eine einprozentige Veränderung des Nettostundenlohns im Durchschnitt zu einer Veränderung von 0,07 \% der gearbeiteten Stunden von Männern und 0,43 \% (0,34\%) von Frauen führt, beides unter der Annahme, dass alle anderen Faktoren unverändert bleiben. Das Resultat, dass das Arbeitsangebot von Frauen wesentlich empfindlicher auf die Veränderung von Löhnen reagiert, kann teilweise durch die Tatsache erklärt werden, dass diese im Durchschnitt immer noch einer viel höheren Belastung durch unbezahlte Arbeit im Haushalt ausgesetzt sind als Männer (OECD 2012e, S. 73). Nach Angaben der OECD ist die Wahrscheinlichkeit, dass Frauen in Teilzeit arbeiten, in Ländern mit hohen Kinderbetreuungskosten höher (ebd., S. 84).

Außerdem weisen Alvaredo et al. $(2013$, S. 9) darauf hin, dass das in der Optimalsteuerliteratur verwendete Lohnfindungsmodell oft extrem vereinfacht ist. Sie berücksichtigen die Möglichkeit, dass die wachsende Verhandlungsmacht der Top-Verdiener diesen hilft, ihre Entlohnung auf Kosten anderer Einkommensgruppen zu erhöhen. Aus dieser Perspektive stellen niedrigere Spitzensteuersätze einen Anreiz zur Erhöhung der Verhandlungsbemühungen dar. Höhere Spitzeneinkommen können somit das Ergebnis der Umverteilung zwischen Einkommensgruppen anstatt Ausdruck zusätzlicher wirtschaftlicher Leistung sein. In diesem Fall läge ein Marktversagen vor, das durch höhere Spitzensteuersätze teilweise korrigiert werden könnte. Wird also die Wirkung von Spitzensteuersätzen auf die Verhandlungsbemühungen berücksichtigt, kann es Spielraum für einen höheren Grenzsteuersatz geben, weil geringere Verhandlungsbemühungen positive Auswirkungen auf die gesamtwirtschaftliche Effizienz haben können.

Obwohl oft argumentiert wird, dass Steuern auf Kapitalerträge die Ersparnisbildung hemmen und somit auch Investitionen und Wachstum, bietet die ökonomische Theorie keine klaren Ergebnisse, die diese Ansicht unterstützen. Dies ist nicht erstaunlich, da auch in einem sehr einfachen Konsummodell der Effekt der Besteuerung auf die Ersparnisse unbestimmt ist (Salanié 2011, S. 289). Banks und Diamond (2010) haben verschiedene Versionen von Modellen bewertet, welche üblicherweise in der optimalen Steuertheorie angewendet werden und bei denen der optimale Steu-

6 Der Fairness halber sollte erwähnt werden, dass der IWF eine Erhöhung der Einkommensbesteuerung zumindest weitgehend diskutiert und nicht per se ausschließt (IWF 2013, S. 33).

7 Vgl. Godar/Truger (2015c) für einen ausführlichen Überblick.

8 Es sei darauf hingewiesen, dass eine Senkung von Arbeitsangebot und Ersparnis nur aus einer einfachen neoklassischen Logik heraus eindeutig als schädlich einzustufen ist, wenn man unterstellt, dass man sich im langfristigen Vollbeschäftigungsgleichgewicht befindet (vgl. Atkinson 1993). Außerhalb dieses Zustandes kann eine Senkung des Arbeitsangebotes auch zu einer Senkung der unfreiwilligen Arbeitslosigkeit führen, und eine Senkung der Ersparnis kann über eine Zunahme des privaten Konsums zu höherem Wachstum führen (siehe hierzu Abschnitt 5). 
ersatz auf Kapitaleinkommen gleich Null ist. Sie kritisieren, dass die herkömmlichen Ergebnisse auf restriktiven Annahmen basieren und bewerten sie daher als nicht robust genug für die politische Analyse. Aus ihrer Sicht kann die Literatur nur wenig über den optimalen Steuersatz für Kapitalerträge sagen.

Anstatt auf eine Steuer mit realen Verhaltensanpassungen zu reagieren, können wohlhabende Haushalte auch einfach versuchen, die Steuer zu vermeiden. Dies kann beispielsweise dadurch geschehen, dass sie in ein Steuerparadieses ziehen ${ }^{9}$ oder ein Bankkonto in einem Steuerparadies eröffnen, wobei ihnen komplizierte rechtliche Strukturen dabei helfen, ihr tatsächliches Vermögen zu verbergen. Henry (2012, S. 36) zufolge liegt der aktuelle Wert der Offshore-Finanzanlagen zwischen 21 und 32 Bio. US-Dollar. Hollingshead (2010) dagegen schätzt die derzeitigen Gesamteinlagen von Nichtansässigen in Steueroasen auf knapp unter 10 Bio. US-Dollar, während Zucman (2014, S. 54) von einer Minimalschätzung von 5,8 Bio $€$ ausgeht.

Selbst wenn man den geringsten Wert ansetzt, stellen Steuerplanung und Steuerflucht offensichtlich eine gewisse Gefahr für eine effektive steuerliche Politik der Umverteilung von Einkommen und Vermögen dar. Piketty et al. (2011) schätzen eine durchschnittliche langfristige Elastizität der Spitzeneinkommen in Bezug auf den Prozentsatz des nach Besteuerung verbleibenden Einkommens („netof-tax rate") von etwa 0,3 bis $0,4 .{ }^{10} \mathrm{Um}$ den optimalen Spitzensteuersatz zu berechnen, haben sie ein Modell entwickelt, welches drei verschiedene Komponenten der Gesamtelastizität integriert: einen angebotsseitigen Effekt (reale Verhaltensanpassungen), einen Steuervermeidungseffekt und einen ausgleichenden (effizienzsteigernden) Effekt auf die Verhandlungsanstrengungen der Top-Verdiener. Für die Vereinigten Staaten schätzen sie, dass der aktuelle Spitzensteuersatz deutlich unter dem optimalen Steuersatz liegt, der trotz gewisser Ausweichreaktionen ein maximales Aufkommen erzielen würde (ebd.). Auch Diamond und Saez (2011, S. 171) zeigen, dass der Spitzensteuersatz in den Vereinigten Staaten von $42,5 \%$ nur dann optimal wäre, wenn die Elastizität der Steuerbasis 0,9 ist. Das ist viel höher als der mittlere Schätzwert von 0,25, den man aus der empirischen Literatur herleiten kann. Mit einem ähnlichen Ansatz hat der IWF (2013, S. 34 ff.) eine Spannweite von aufkommensmaximierenden Spitzensteuersätzen für 16 OECDLänder berechnet. In zwölf Ländern liegt der tatsächliche Spitzensteuersatz unterhalb oder in der unteren Hälfte dieser Spannweite, was einen erheblichen Spielraum für höhere Steuersätze anzeigt.

\subsection{Negative Wirkungen auf die Unternehmensentscheidungen?}

Weit verbreitet ist die Behauptung, hohe Unternehmenssteuersätze veranlassten die Unternehmen dazu, ihre Produktion ins Ausland zu verlagern und damit die Beschäftigung im Inland zu verringern, weshalb Unterneh- menssteuern häufig als besonders wachstumsschädlich angesehen werden (OECD 2010, S. 20). Der theoretische Mechanismus hinter diesen Effekten liegt in der Wirkung der Körperschaftssteuer auf die Kapitalkosten: Die neoklassische Idee ist, dass Unternehmen Kapital akkumulieren, solange die Rentabilität von Investitionen die zu ihrer Finanzierung notwendigen Kapitalkosten übersteigt (de Mooij/Ederveen 2008, S. 684). Wie sich herausstellt, beruht dieser Standardansatz jedoch auf sehr engen theoretischen Annahmen. Die Tatsache, dass die Unternehmen solange investieren, wie es rentabel ist, also die Renditen höher als die Kapitalkosten sind, bietet keine Antwort auf die Frage, wie viel höher die Renditen sein müssen. Bei der Betrachtung wird impliziert, dass Unternehmen keinen Gewinn bei ihrem marginalen - d. h. letzten noch rentablen - Investitionsprojekt realisieren. Doch unter den Bedingungen eines unvollkommenen Marktes erzielen die Unternehmen auch bei ihrem marginalen Investitionsprojekt mehr als den unterstellten Nullgewinn. Dadurch gibt es immer noch einen Anreiz zu investieren, solange die Unternehmenssteuer den ökonomischen Gewinn nicht vollständig absorbiert.

Außerdem weisen Musgrave und Musgrave (1989, S. 306) darauf hin, dass die Auswirkungen von Unternehmenssteuern von der zugrunde liegenden Investitionstheorie abhängen. Investitionen mögen zwar unter sonst gleichen Bedingungen über ihre Wirkung auf die Kapitalkosten negativ von der Besteuerung abhängen. Es gibt jedoch noch sehr viele andere Determinanten der Investitionstätigkeit, wie zum Beispiel vergangene Umsatzerlöse, das Geschäftsklima und die Lohnstückkosten. Darüber hinaus sollten die potenziell positiven langfristigen Effekte der öffentlichen Finanzierung von F\&E-Ausgaben und die Bildung von $\mathrm{Hu}$ mankapital berücksichtigt werden, ebenso wie auch die möglicherweise positiven Agglomerationseffekte, welche die negativen Auswirkungen der Besteuerung kompensieren können (Brühlhart et al. 2012).

Empirische Analysen zeigen, dass das Investitionsverhalten durch die Unternehmensbesteuerung beeinflusst wird, aber es ist schwer, verlässliche Schätzungen für die Größe und damit die Relevanz dieser Effekte zu bekommen. Es gibt nicht viele empirische Studien zu den Effekten der Besteuerung auf die aggregierten realen Investitionen. Die Resultate mikroökonomischer Studien deuten auf negative Auswirkungen der Besteuerung auf die Investitionen hin. Die Spanne reicht von eher unelastischen $(-0,25)$ zu eher elastischen (-1) Reaktionen der Investitionen. Es ist

9 Trotz einzelner Beispiele für Migration von Millionären fin den Kleven et al. (2010) sowie Young und Varner (2011) nur eine sehr schwache bis keine statistische Korrelation zwischen der Wahl des Wohnsitzes von Millionären und dem Steuerrecht.

10 Das bedeutet, dass eine zehnprozentige Verminderung der net-of-tax-rate nur zu einer drei- bis vierprozentigen Verminderung des zu versteuernden Einkommens führt. 
aber schwierig, diese Ergebnisse auf die aggregierten Investitionen der gesamtwirtschaftlichen Ebene zu übertragen (Hanlon/Heitzman 2010, S. 148). Eine Meta-Studie von de Mooij und Ederveen (2008) über die Auswirkungen der Besteuerung auf ausländische Direktinvestitionen zeigt sehr unterschiedlich starke Auswirkungen: Im Durchschnitt reduziert eine Steuererhöhung um einen Prozentpunkt an einem bestimmten Ort das ausländische Kapital um 3,3 \% (ebd., S. 689). Allerdings ist die Standardabweichung von 4,4 sehr hoch und die ausländischen Direktinvestitionen können nicht als Proxy für die aggregierten realen Investitionen verwendet werden, da sie auch Portfolioinvestitionen beinhalten. Zwei aktuelle Studien haben die Investitionseffekte der Unternehmenssteuersenkungen in Deutschland (Reinhard/Li 2011) und Großbritannien (Maffini 2013) untersucht und kamen zu dem ernüchternden Ergebnis, dass es keine überzeugenden Belege für eine investitionssteigernde Wirkung gibt.

Neben den realen Verhaltensanpassungen sind die Vermeidungsstrategien der Unternehmen, die die Steuerbasis manipulieren, ohne die tatsächliche wirtschaftliche Aktivität in einem Land zu reduzieren oder zu steigern, ein viel diskutiertes Thema. Nach einem umfassenden Bericht der OECD (2013b) über die Erosion der Steuerbasis und die Gewinnverlagerung gibt es mehrere Möglichkeiten für Unternehmen, die Einkommen auf dem Papier zwischen ihren unterschiedlichen Unternehmensteilen und -standorten hinund herzuschieben und dabei in Länder mit niedrigeren Steuersätzen oder speziellen Ausnahmen zu verlagern. Solche Möglichkeiten ergeben sich beispielsweise durch die Nutzung von Lizenzen für Marken, Patente oder bestimmten Finanzdienstleistungen, die durch eine Tochtergesellschaft in einem Niedrigsteuerland erbracht werden oder durch die Manipulation von Verrechnungspreisen. Zwar gibt es keine verlässlichen Daten darüber, wie viel Gewinnverlagerung tatsächlich stattfindet (OECD 2013b, S. 15), die Existenz solcher Aktivitäten ist aber weitgehend unbestritten.

Heckemeyer und Overesch (2013) haben die empirische Literatur zu den Gewinnverlagerungsaktivitäten multinationaler Unternehmen untersucht. Die 25 Studien schätzen im Durchschnitt eine Semi-Elastizität der ausgewiesenen Gewinne bzw. der Gewinne vor Zinsen und Steuern in Bezug auf das internationale Steuergefälle zwischen einem Staat und den Standorten von Zweigunternehmen von 1,55, mit einer relativ hohen Standardabweichung von 2,23 (ebd., S. 8). Obwohl die Zahl auf den ersten Blick erheblich scheint, bedeutet dies im Durchschnitt, dass ein Land mit einem Gesamtsteuersatz auf Unternehmensgewinne von $20 \%$ diesen um fünf Prozentpunkte bzw. um ein Viertel erhöhen kann und dafür lediglich 7,75\% der Steuerbasis verliert. Daher können aufgrund der Steuervermeidungsstrategien zwar nicht die vollen zusätzlichen Einnahmen aus der Steuererhöhung angesetzt werden, aber immerhin mehr als zwei Drittel davon.

Alles in allem stellt sich heraus, dass die ökonomischen Argumente gegen progressive Besteuerung wesentlich schwächer sind als zumeist behauptet. Aus theoretischer und empirischer Sicht müssen die negativen Auswirkungen auf Wachstum und Beschäftigung sowie die Erosion der Steuerbasis nicht groß sein, wobei gerade die jüngere Literatur diese Einschätzung tendenziell besonders unterstützt (Bach 2014). Außerdem scheinen andere Einflussfaktoren (Konjunktur, Infrastrukturinvestitionen, Forschungs- und Entwicklungsausgaben, das Bildungssystem) viel wichtiger zu sein. Wenn das Aufkommen aus der höheren progressiven Besteuerung zur Stärkung dieser Faktoren mittels höherer Staatsausgaben verwendet wird, kann die gesamtwirtschaftliche Wirkung insgesamt sogar durchaus positiv sein. Mittlerweile kommen auch IWF-Autoren zu dem Ergebnis, dass es keine negative Korrelation zwischen Umverteilung und Wachstum gibt (Ostry et al. 2014).

\section{Makroökonomische Argumente zugunsten progressiver Besteuerung}

Die Argumente für eine progressivere Besteuerung können aus einer makroökonomischen Perspektive noch weiter gestärkt werden, sofern die Wirtschaft unter unzureichender aggregierter Nachfrage leidet. ${ }^{11}$ Aktuelle Multiplikatorschätzungen bestärken die traditionelle keynesianische These, dass die Finanzpolitik wirksam ist, vor allem unter den aktuellen Bedingungen in der Eurozone, in der die Geldpolitik an der Nullzinsgrenze angelangt ist (Auerbach/Gorodnichenko 2013; Batini et al. 2012). Wie die keynesianischen Lehrbuchmodelle und das Haavelmo-Theorem nahelegen, scheinen in der empirischen Literatur die ausgabenseitigen Multiplikatoren größer als die einnahmeseitigen zu sein (Gechert 2013; Gechert/Rannenberg 2014). Dies deutet darauf hin, dass eine Finanzierung zusätzlicher Staatsausgaben über progressive Steuern förderlich für Wachstum und Beschäftigung ist.

Darüber hinaus gibt es auch eine gesamtwirtschaftliche Begründung für aufkommensneutrale Steuerreformen, die eine Umverteilungswirkung als Ziel haben. Wenn die Ungleichheit negativ auf den privaten Konsum wirkt, kann eine Erhöhung des Progressionsgrades der Besteuerung über die resultierende Zunahme des privaten Konsums Wachstum und Beschäftigung stärken. Keynes (1973 [1936], Kap. 2; 1937, S. 219ff.) selbst betonte in seinen Ausführungen zwar besonders die Bedeutung der Investitionsnachfrage, weil er deren starke Volatilität in Kombination mit dem Multiplikator-Prozess für die wichtigste Ursache von Schwankungen der gesamtwirtschaftlichen Aktivität hielt. Die Investitionsnachfrage hänge von den schwankenden subjektiven Erwartungen der Unternehmen hinsichtlich der Profitabilität von realwirtschaftlichen Investitionen und dem monetären Zins ab, der wiederum von der ebenfalls schwankenden Liquiditätspräferenz der Wirtschafts-

11 Vgl. Godar et al. (2015) für einen ausführlichen Überblick. 
subjekte beeinflusst werde. Eine zentrale Rolle spielen in Keynes' Argumentation jedoch auch der private Konsum und vor allem die Tatsache, dass dieser als einkommensabhängig betrachtet wird. Keynes geht davon aus, dass der private Konsum positiv vom gesamtwirtschaftlich verfügbaren Einkommen abhängt, wobei die marginale Konsumneigung angibt, wie groß der Teil einer Einkommenserhöhung ist, der in zusätzlichen Konsum fließt und damit automatisch auch, wie groß der Teil ist, der in die Ersparnis fließt. Komme es aufgrund eines Anstiegs der Investitionstätigkeit zu einem Anstieg des gesamtwirtschaftlichen Einkommens, dann führe dies gemäß der marginalen Konsumneigung zu einem zusätzlichen Anstieg des privaten Konsums, der wiederum einen zusätzlichen Einkommensanstieg verursacht etc. Der ausgelöste Multiplikator-Prozess werde umso stärker ausfallen, je höher die marginale Konsumneigung und je geringer die marginale Sparneigung spiegelbildlich hierzu sei.

Unter Zugrundelegung dieser theoretischen Zusammenhänge lässt sich offensichtlich ein negativer Zusammenhang zwischen dem Grad der Ungleichheit in der Einkommensverteilung und dem privaten Konsum herstellen: Wenn Haushalte mit geringem Einkommen eine höhere Konsumneigung als Haushalte mit höherem Einkommen haben, dann führt eine Umverteilung zugunsten der einkommensschwachen Haushalte zu einer höheren gesamtwirtschaftlichen Konsumneigung und damit zu einem Anstieg des privaten Konsums. In diesem Fall würde eine (steuerpolitische) Korrektur der Disparität in der Einkommensverteilung zu einer Stärkung des privaten Konsums und damit ceteris paribus einer Zunahme von Wachstum und Beschäftigung führen.Daher kann von einer Steigerung der Konsumausgaben durch eine steuerlich induzierte Verringerung der Einkommensungleichheit ausgegangen werden. ${ }^{12}$

Damit stellt sich die Frage, unter welchen Bedingungen sich eine solche Nachfrageerhöhung auch tatsächlich in eine höhere gesamtwirtschaftliche Aktivität transformiert. Offenkundig hängt die Antwort auf diese Frage sehr stark vom zugrunde gelegten makroökonomischen Paradigma ab. Dem gegenwärtig die Literatur dominierenden Ansatz des „New Consensus“ (Clarida et al. 1999) zufolge wird die höhere gesamtwirtschaftliche Aktivität wahrscheinlich nur kurzfristig auftreten. Auf lange Sicht würden die sogenannte NAIRU (non accelerating inflation rate of unemployment) und das zugehörige Beschäftigungs- und Output-Gleichgewicht vorherrschen und die kurzfristigen Auswirkungen auf die Beschäftigung auslöschen. Das NCM („,new consensus in macroeconomics")-Modell lässt sich jedoch durch einige Modifikationen schrittweise postkeynesianischen makroökonomischen Ansätzen annähern, die ganz in der skizzierten Tradition von Keynes (1973 [1936]; 1937) kurz- wie langfristig der aggregierten Nachfrage eine wichtige Rolle für Wachstum und Beschäftigung zuweisen. In diesen Ansätzen, die durch die erhebliche Erschütterung der StandardNCM-Modelle und ihrer restriktiven Annahmen durch die
Große Rezession sicherlich erheblich an Überzeugungskraft gewonnen haben, führt eine Stärkung der Umverteilung über Steuern also tendenziell auch zu einer Stärkung von Wachstum und Beschäftigung. Damit kann der Trade-off zwischen Verteilungsgerechtigkeit und Effizienz aus gesamtwirtschaftlicher Sicht auch langfristig aufgelöst werden.

\section{Steuerpolitische Schlussfolgerungen}

Die Aussichten für eine progressive Reform des Steuersystems haben sich in den letzten Jahren recht günstig entwickelt. ${ }^{13}$ Es gibt einige Anzeichen dafür, dass der Abwärtstrend bei der progressiven Besteuerung seit der Krise zum Stillstand gekommen ist. Seither hat eine Reihe von internationalen Institutionen sich in mehr oder weniger fortschrittlicher Art und Weise für sozialverträgliche Steuerreformen zum Zwecke der Haushaltskonsolidierung ausgesprochen.

Auf internationaler Ebene sollte der breite Konsens über die Notwendigkeit der Bekämpfung von Steuerhinterziehung, die Begrenzung der Steuervermeidung und die Einführung einer Finanztransaktionssteuer zur möglichst ehrgeizigen Umsetzung entsprechender Reformen genutzt werden. Die Überarbeitung der EU-Zinsrichtlinie mit dem Ziel, dass über Zinseinkommen hinaus auch Dividenden, Gewinne und alle anderen Formen von Kapitaleinkommen erfasst werden (Europäischer Rat 2014) ist ein wichtiger Schritt gegen die Steuerhinterziehung von natürlichen Personen. Das Gleiche gilt für den von der OECD und den G20 angestrebten globalen Informationsaustausch zwischen den Steuerbehörden. (OECD 2014b, S. 3). Im Bereich der Unternehmensbesteuerung bildet der Aktionsplan der OECD (2013c) bezüglich der Erosion der Steuerbasis und der Gewinnverlagerung einen wichtigen Vorstoß. Ein möglicherweise noch wichtigerer Schritt wäre der „Unitary Taxation-Ansatz“, nach dem multinationale Unternehmen ihre weltweite Konzernbilanz bei den lokalen Steuerbehörden einreichen müssen, sodass ihre internen Transfers nicht mehr von Interesse wären (Picciotto 2012). Um schädlichen Steuerwettbewerb auszuschalten, müsste dieser allerdings um Mindeststeuersätze ergänzt werden. Eine weltweite Vermögensteuer, wie sie von Piketty (2014, Kapitel 15) vorgeschlagen wurde, könnte das Ziel für die internationale Besteuerung extrem reicher

12 Allerdings sind die zugrunde liegenden Hypothesen zum privaten Konsumverhalten sicherlich nicht unumstritten (siehe vanTreeck und Sturn 2012, S. 13ff.).

13 Für eine ausführlichere Diskussion der Reformvorschläge und Alternativen siehe Godar/Truger (2015b), im Allgemei nen und für den Fall Deutschland insbesondere EickerWolf/Truger (2014) 
Haushalte sein. Eine Finanztransaktionssteuer, die sowohl Aktien als auch Derivative abdeckt, könnte die Volatilität der Finanzmärkte reduzieren und dabei gleichzeitig beträchtliche Einnahmen generieren (Schulmeister et al. 2008). Leider gibt es bei all diesen Vorschlägen die ernsthafte Gefahr, dass sie durch politischen Druck verzögert, verwässert oder gar nicht umgesetzt werden.

Unabhängig vom Erfolg der Maßnahmen auf internationaler Ebene sollte die nationale Politik daher die Spielräume für eine progressivere Besteuerung entschlossen nutzen. Es wurde gezeigt, dass es auf nationaler Ebene erheblichen Spielraum für steuerliche Umverteilung gibt. Daher besteht keine Notwendigkeit, sich auf die von den internationalen Institutionen vorgeschlagenen Reformen, wie die Ausweitung der Bemessungsgrundlage und die Erhöhung der Besteuerung von Wohneigentum, zu beschränken. Stattdessen scheint es für viele nationale Regierungen erheblichen Spielraum zur Erhöhung des Spitzensteuersatzes der Einkommensteuer, der Unternehmenssteuersätze und der Besteuerung von Kapital und Vermögen im Allgemeinen zu geben. Die nationalen Regierungen sollten diese Möglichkeiten ausschöpfen, weil es einerseits zusätzliche Einnahmen bringt, die für öffentliche Zwecke verwendet werden können, und andererseits zur Verringerung der Ungleichheit beiträgt.

\section{LITERATUR}

Alvaredo, F./Atkinson, A./Piketty, T./Saez, E. (2013): The top 1 percent in international and historical perspective, in: Journal of Economic Perspectives 27 (3), S. 3-20

Atkinson, A. B. (1993): Introduction, in: Atkinson, A. B./Mogensen, G. V. (Hrsg.): Welfare and work incentives. A north European perspective, Oxford

Auerbach, A. J./Gorodnichenko, Y. (2013): Fiscal multipliers in recession and expansion, in: Alesina, A./Giavazzi, F. (Hrsg.): Fiscal policy after the financial crisis, Chicago

Bach, S. (2014): „Reichensteuer"-Diskussion: Hintergrund und Perspektiven: Deutsches Institut für Wirtschaftsforschung, DIW Roundup, Politik im Fokus (16), Berlin

Banks, J./Diamond, P. (2010): The base for direct taxation. Prepared for the Report of a Commission on Reforming the Tax System for the 21st Century, Institute for Fiscal Studies, www.ifs.org.uk/mirrleesreview

Batini, N./Callegari, G./Melina, G. (2012): Successful austerity in the United States, Europe and Japan, IMF Working Paper 12/190, Washington DC Brühlhart, M./Jametti, M./Schmidheiny, K. (2012): Do agglomeration economies reduce the sensitivity of firm location to tax differentials?, in: The Economic Journal 122 (563), S. 1069-1093

Clarida, R./Gali, J./Gertler, M. (1999): The science of monetary policy: A new Keynesian perspective, in: Journal of Economic Literature 37 (4),

S. $1661-1707$

De Mooij, R./Ederveen, S. (2008): Corporate tax elasticities: A reader's guide to empirical findings, in: Oxford Review of Economic Policy 24 (4), S. 680-697 Deloitte (2013): International Tax. Highlights, for different countries, https://dits.deloitte.com/\#TaxGuides

Devereux, M./Griffith, R./Klemm, A. (2004): Why has the UK corporation tax raised so much revenue?: The Institute for Fiscal Studies WP04/04, London Diamond, P./Saez, E. (2011): The case for a progressive tax: From basic research to policy recommendations, in: The Journal of Economic Perspectives 25 (4), S. 165-190

Eicker-Wolf, K./Truger, A.(2014): Demystifying a 'shining example': German public finances under the debt brake: Unmasking the model pupil, in: Dullien, S./ Hein, E./Truger, A. (Hrsg.): Makroökonomik, Entwicklung und Wirtschaftspolitik. Festschrift für Jan Priewe, Marburg, S. 369-391

European Trade Union Confederation (ETUC) (2010): ETUC Resolution on the Economic Crisis: New Sources of Finance, http://www.etuc.org//MG/pdf Resolution-The-eco-crisis-New-sources-financeEN.pdf

European Attac Network (2013): For a Europe-wide coordinated levy on wealth, http://www.attac.org/sites/default/files/EAN-Wealth-Levy-Concept.pdf
Europäische Kommission (2012): Taxation trends in the European Union. Data for EU member states, Iceland and Norway, Eurostat Statistical Books Europäische Kommission (2013a): Taxation trends in the European Union, Eurostat Statistical Books

Europäische Kommission (2013b): Fighting tax evasion: Commission proposes widest scope of automatic exchange of information within the EU", Press Release IP/13/530. Brussels, 12 June, http://europa.eu/rapid/press-release IP-13-530_en.htm.

Europäische Kommission (2013c): Taxation of the financial sector. Taxation and customs Union, http://ec.europa.eu/taxation_customs/taxation/other_taxes/ financial sector/ (letzter Zugriff 16.09.2013)

Europäische Kommission (2014): Annual macroeconomic database, November Europäischer Rat (2012): European Council conclusions, 1-2 March,

EUCO 4/1/12 REV 1, Brussels, http://register.consilium.europa.eu/pdf/en/12/ st00/st00004-re01.en12.pdf

Europäischer Rat (2014): Richtlinie 2014/48/EU des Rates vom 24. März zur Änderung der Richtlinie 2003/48/EG im Bereich der Besteuerung von Zinserträgen, Amtsblatt der Europäischen Union, 15. April, L 111/51

Evers, M./de Mooij, R./van Vuuren, D. (2008): The wage elasticity of labour supply: A synthesis of empirical estimates, in: De Economist 156 (1), S. 25-43 Gechert, S. (2013): What fiscal policy is most effective? A meta-regression analysis, IMK Working Paper (117), September, Düsseldorf

Gechert, S./Rannenberg, A. (2014): Are fiscal multipliers regime-dependent? A meta regression analysis, IMK Working Paper (139), September, Düsseldorf Godar, S./Truger, A. (2015a): Goals of taxation and taxation trends in the OECD since the 1980s: Traditional standards of tax justice under pressure: Global Labour University Working Paper (im Erscheinen)

Godar, S./Truger, A. (2015b): Standard arguments against progressive taxation: A critical evaluation, Global Labour University Working Paper (im Erscheinen)

Godar, S./Truger, A. (2015c): Progressive tax reform in OECD countries: Recent proposals and perspectives, Global Labour University Working Paper (im Erscheinen)

Godar, S./Paetz, C./Truger, A. (2015): The scope for progressive tax reform in the OECD countries: A macroeconomic perspective with a case study for Germany, in: Revue de I'OFCE 138 (1) (im Erscheinen)

Hanlon, M./Heitzman, S. (2010): A review of tax research, in: Journal of Accounting and Economics 50 (2-3), S. 127-178

Heckemeyer, J./Overesch, M. (2013): Multinationals' profit response to tax differentials: Effect size and shifting channels, ZEW Discussion Paper 13-045, http://ftp.zew.de/pub/zew-docs/dp/dp13045.pdf

Hein, E. (2008): Money, distribution conflict and capital accumulation, Contributions to "monetary analysis", Basingstoke

Henry, J. (2012): The price of offshore revisited. New estimates for 'missing' global private wealth, income, inequality, and lost taxes, Tax Justice Network, http://www.taxjustice.net/cms/upload/pdf/Price_of_Offshore_Revisited_120722. pdf

Hollingshead, A. (2010): Privately held, non-resident deposits in secrecy jurisdictions, Global Financial Integrity, http://www.gfintegrity.org/storage/gfip/ documents/reports/gfi_privatelyheld_web.pdf.

Internationaler Währungsfonds (IWF) (2013): Fiscal monitor 2013: Taxing times, Washington DC

International Trade Union Confederation (ITUC) (2010): Global unions statement to the G20, http://www.ituc-csi.org/IMG/pdf/01-tuacG8G20-Seoul_ English.pdf

International Labour Organization (ILO) (2011): World of work report 2011: Making markets work for jobs, International Labour Office, Geneva, http://www.ilo.org/wcmsp5/groups/public/@dgreports/@dcomm/@publ/ documents/publication/wcms_166021.pdf

Keynes, J. M. (1973 [1936]): The general theory of employment, interest, and money, in: Johnson, E./Moggridge, D. (Hrsg.): The collected writings of J.M. Keynes, Vol. 7, London

Keynes, J. M. (1937): The general theory of employment: in: Quarterly Journal of Economics 51 (2), S. 209-223

Kleven, H./Landais, C./Saez, E (2010): Taxation and international migration of superstars: Evidence from the European football market, NBER Working Paper (16545), http://www.nber.org/papers/w16545

Lavoie, M. (2009): Taming the new consensus: Hysteresis and some other post-Keynesian amendments, in: Fontana, G./Setterfield, M. (Hrsg.): Macroeconomics and macroeconomic pedagogy, Basingstoke 
Maffini, G. (2013): Corporate tax policy under the labour government, 1997-2010, in: Oxford Review of Economic Policy 29 (1), S. 142-164 Musgrave, R./Musgrave, P. (1989): Public finance in theory and practice, New York

Organisation for Economic Co-operation and Development (OECD) (2010): Tax policy reform and economic growth, OECD Tax Policy Studies (20), OECD Publishing

OECD (2011): Divided we stand: Why inequality keeps rising, OECD Publishing OECD (2012a): Revenue Statistics 2012, OECD Publishing

OECD (2012b): Taxing wages 2011, OECD Publishing

OECD (2012c): Income inequality and growth: The role of taxes and transfers, OECD Economics Department Policy, Notes (9)

OECD (2012d): OECD's current tax agenda 2012, OECD Publishing

OECD (2012e): Gender equality in education, employment and entrepreneurship: Final report to the MCM 2012, http://www.oecd.org/

employment/50423364.pdf

OECD (2013a): Tax database. Overall statutory tax rates on dividend income/ top income rates, OECD Publishing

OECD (2013b): Addressing base erosion and profit shifting, OECD Publishing OECD (2013c): Action plan on base erosion and profit shifting, OECD Publishing

OECD (2014a): Revenue statistics 2014, OECD Publishing

OECD (2014b): Automatic exchange of financial account information, Background Information Brief, 29 October

Ostry, J./Berg, A./Tsangarides, C. (2014): Redistribution, inequality and growth, IMF Staff, Discussion Note 14/02, Washington DC

Picciotto, S. (2012): Towards unitary taxation of transnational corporations, Tax Justice Network, http://www.taxjustice.net/cms/upload/pdf/Towards Unitary_Taxation_1-1.pdf

Piketty T. (2014): Capital in the twenty-first century, Cambridge

Piketty, T./Saez, E./Stantcheva, S. (2011): Optimal taxation of top labor incomes: A tale of three elasticities, NBER Working Paper (17616), http://www.nber. org/papers/w17616

Piketty, T./Zucman, G. (2013): Capital is back: Wealth-income ratios in rich countries 1700-2010, http://piketty.pse.ens.fr/files/PikettyZucman2013WP.pdf Reinhard, L./Li, S. (2011): The influence of taxes on corporate financing and investment decisions against the background of the German tax reforms, in: The European Journal of Finance 17 (7-8), S. 717-737

Rosen, H./Gayer, T. (2008): Public finance, New York

Salanié, B. (2011): The economics of taxation, Cambride, MA

Schratzenstaller, M. (2004): Towards dual income taxes - a country comparative perspective, in: ifo Institut für Wirtschaftsforschung, CESifo DICE Report 2 (3), S. 23-30

Schulmeister, S./Schratzenstaller, M./Picek, O.(2008): A general financial transaction tax. Motives, revenues, feasibility and effects, Research Study by the Austrian Institute of Economic Research,

http://www.wifo.ac.at/jart/prj3/wifo/resources/person_dokument/person_ dokument.jart?publikationsid=31819\&mime_type=application/pdf

Spengel, C./Elschner, C/Endres, D. (2012): Effective tax levels using the Devreux/Griffith Methodology, Project for the EU Commission TAXUD/2008/ CC/099, Final Report 2012, Mannheim

Tax Justice Network (2013): World Forum declaration on tax justice, March, Tunis, http://taxjustice.blogspot.de/2013/04/world-forum-declaration-on-taxjustice.html

United Nations Conference on Trade and Development (UNCTAD) (2012):

Trade and development report 2012. Policies for inclusive and balanced growth, Geneva, http://unctad.org/en/PublicationsLibrary/tdr2012_en.pdf

Van Treeck, T./Sturn, S. (2012): Income inequality as a cause of the Great Recession? A survey of current debates: International Labour Organization, Conditions of work and employment series (39), Geneva

Young, C./Varner, C. (2011): Millionaire migration and state taxation of top incomes. Evidence from a natural experiment, in: National Tax Journal 64 (2), S. $255-284$

Zucman, G. (2014): Steueroasen. Wo der Wohlstand der Nationen versteckt wird, Berlin

\section{AUTOREN}

SARAH GODAR, M.A., hat im Herbst 2014 ihr Masterstudium "International Economics" an der Hochschule für Wirtschaft und Recht (HWR) Berlin abgeschlossen und war freie Mitarbeiterin im von der Hans-Böckler-Stiftung finanzierten Projekt „Combating Inequality“. Arbeitsschwerpunkte: Steuerpolitik, Verteilung und Makroökonomik.

CHRISTOP PAETZ, M.A., hat im Herbst 2014 sein Masterstudium "International Economics" an der HWR Berlin abgeschlossen und war freier Mitarbeiter im von der Hans-Böckler-Stiftung finanzierten Projekt "Combating Inequality". Arbeitsschwerpunkte: Finanzwissenschaft, Staatsverschuldung und europäische Wirtschaftspolitik.

ACHIM TRUGER, Dr. rer.pol., ist Professor für Volkswirtschaftslehre, insbesondere Makroökonomie und Wirtschaftspolitik, an der HWR Berlin sowie Senior Research Fellow am Institut für Makroökonomie und Konjunkturforschung (IMK) der Hans-Böckler-Stiftung. Arbeitsschwerpunkte: Makroökonomie und Wirtschaftspolitik, Steuer- und Finanzpolitik.

achim.truger@hwr-berlin.de 